\title{
A Software Framework for Mixed Finite Element Programming
}

\author{
Hans Petter Langtangen and Kent-Andre Mardal \\ Simula Research Laboratory \\ and \\ Dept. of Informatics, University of Oslo \\ $\{\mathrm{hpl}$, kent-and\}@simula.no
}

\begin{abstract}
Many code developers think the implementation of mixed finite elements on general unstructured meshes is challenging. This assertion is reflected in the many efforts on constructing stabilized finite element methods (e.g. for flow problems), where standard elements can be used, and the fact that there are very few libraries offering a mixed finite element programming environment. In this paper we described a software framework for mixed finite element programming, which makes the use of mixed methods as easy as standard finite element methods. The various abstractions used in this framework will be explained. The tools are made available in the generic Diffpack software.
\end{abstract}

\section{Introduction}

Mixed finite elements refers to solving a system of partial differential equations (PDEs) using different finite elements for the different unknown functions. For example, when solving the Navier-Stokes equations for incompressible viscous fluid flow, a standard finite element method, where the pressure and velocity components are approximated using the same elements, will normally not work well. A stable and robust discretization can be achieved when the pressure and velocities are approximated by different elements, one example being quadratic elements for the velocities and linear elements for the pressure. Similarly, when solving the Poisson equation $-\nabla \cdot(k \nabla u)=f$ as a system of equations,

$$
\nabla \cdot \boldsymbol{q}=f, \quad \boldsymbol{q}=-k \nabla u,
$$

stability of the discretization and optimal convergence rate is ensured by using different elements for $u$ and $\boldsymbol{q}$. The working choices of combination of elements are dictated by the Babuska-Brezzi condition $[1,2,11]$. The great advantage of the system-formulation of Poisson's equation is the possibility to approximate, at a given cost, $\boldsymbol{q}$ with higher accuracy than when applying the conventional finite element method to $-\nabla \cdot(k \nabla u)=f$ and then numerically differentiate $u$ to get $\boldsymbol{q}=-k \nabla u$. In problems where $\boldsymbol{q}$ is of primary interest (e.g., porous media flow, potential flow, torsion problems) the use of mixed finite elements is hence desirable. 
Programming with mixed finite elements needs considerable more bookkeeping than programming with conventional finite elements. This is one reason why there have been numerous attempts to stabilize numerical methods to avoid mixed finite elements. One common approach is to add stabilization terms to the PDEs. This introduces new parameters in the problem that need to be determined experimentally. The advantage of mixed finite elements is that the numerical problem is stable without parameters to be tuned.

Another disadvantage of mixed finite elements is that the resulting linear systems are often indefinite. A typical linear system can be written

$$
\left[\begin{array}{cc}
A & B \\
B^{T} & 0
\end{array}\right]\left[\begin{array}{l}
v \\
p
\end{array}\right]=\left[\begin{array}{l}
f \\
g
\end{array}\right] .
$$

Finding efficient iterative methods and preconditioners for linear systems with this type of coefficient matrices has been a difficult problem. The indefiniteness may cause breakdown of iterative methods like preconditioned Krylov solvers, such as GMRES. GMRES handles indefinite and nonsymmetric matrices, but the preconditioner needs to be constructed properly such that the eigenvalues are not close to zero. A promising remedy for this problem seems to be block preconditioning, which will be described from an implementional point of view later (the mathematical properties are considered in [10]).

A variety of alternative solution algorithms exists (see e.g., [5, 9, 11] for an overview of methods for the Navier-Stokes equations.). Of particular interest is the basic iteration on the pressure Schur complement, considered thoroughly in [11]. It basically consist in eliminating $\boldsymbol{v}$ from (1) to obtain an equation for the pressure,

$$
\boldsymbol{B}^{T} \boldsymbol{A}^{-1} \boldsymbol{B} \boldsymbol{p}=\boldsymbol{B}^{T} \boldsymbol{A}^{-1} \boldsymbol{f}-\boldsymbol{g}
$$

This equation is then solved iteratively with the basic iteration and a preconditioner for $\boldsymbol{B}^{T} \boldsymbol{A}^{-1} \boldsymbol{B}$. Recent research indicates that there is a very close connection between different methods like operator splitting and proper preconditioners for the fully coupled mixed system $[5,11]$. The principle idea for both methods is to explore the block form of the matrix and construct special preconditioners for $\boldsymbol{A}$ and $\boldsymbol{B}^{T} \boldsymbol{A}^{-1} \boldsymbol{B}$. Numerical experiments have shown promising results in particular test cases for both operator splitting algorithms and strategies for the fully coupled system, but the robustness and efficiency in general are still unknown issues.

In addition, it is an open question whether the increased accuracy that can be obtained with mixed finite elements also imply increased computational time because of the lack of efficient linear solvers. If this is the case, conventional finite elements with more degrees of freedom can perhaps deliver the same accuracy at the same CPU cost. Hence it is desirable to have a framework were all methods can be tested in each given application.

Mixed finite elements and associated iterative solution strategies can now be used to construct accurate, efficient, and robust numerical methods for many problems, especially in fluid dynamics applications governed by the NavierStokes equations or the Poisson equation. The major obstacle to increased use of 
mixed finite elements is that the computer programming on general unstructured grids is difficult. In addition, the theory of mixed elements is very abstract, and it is difficult to see how it should be implemented and what is actually gained in practice. In this paper we address software abstractions that make mixed finite element as easy to program with as conventional finite elements. Furthermore, we address software abstractions for easy programming of preconditioners utilizing the block structure of the coefficient matrices. Special emphasis is paid to constructing the software abstractions on basis of the abstractions already present in conventional finite element programs. This makes it possible to add mixed finite element support "on top" of existing codes, at least in principle.

We have implemented the suggested mixed finite element programming tools in the software system Diffpack [3, 4, 6, 7]. Diffpack is a collection of $\mathrm{C}++$ classes organized in libraries. Originally, Diffpack was designed and implemented for conventional finite element methods. The mixed finite element support was added many years later. Writing an application for solving a system of PDEs is typically a matter of

- defining and reading problem-specific parameters,

- defining a finite element grid and associated scalar and vector fields over the grid representing each unknown field in the system of PDEs,

- defining essential boundary conditions,

- defining the integrand in the weak formulation of the problem,

- specifying linear system storage and associated (iterative) solver,

- dumping results (computed fields) to databases for visualization.

An application code is normally just a few pages long. All the numerics that can be shared among different applications take place in generic libraries. The advantage of such a design is that the application codes become short, since the libraries provide the major parts of the the code. Moreover, the applications are more reliable and easier to debug, because a well-tested numerical kernel is reused in many different problems.

Due to the space limitations of this paper we concentrate the exposition on principal ideas rather than on Diffpack-specific implementational details.

\section{Software Abstractions for Mixed Finite Elements}

Conventional finite elements are associated with a grid, which defines the geometry of the domain and elements as well as the basis functions (also called trial functions or shape functions). In particular, the geometry of the elements is described by a mapping of a reference element onto a possible deformed element in the physical coordinates. When the mapped basis functions coincide with the geometry functions, the element is referred to as isoparametric.

In problems solved by mixed finite elements, there will be at least one element that is not isoparametric. For example, in a fluid flow problem with quadratic triangular elements for the velocity components and linear triangular elements for the pressure, the mapping can be based on the quadratic basis functions, 
allowing curved sides in the elements. The velocity elements are then isoparametric, but the pressure elements are not since different basis functions are used for the mapping and for approximating the unknown. The next subsection describes how to deal with a common grid for all unknowns, but allow different unknowns to have different sets of basis functions.

\subsection{Grids for Basis Functions}

We apply a standard finite element grid to describe the geometry of all elements. The basis functions associated with conventional isoparametric elements in this grid are used in the mapping of the reference elements onto elements in the physical coordinate system. These basis functions are referred to as geometry functions.

Each primary unknown over this grid has elements whose geometries are described by the geometry functions, but the unknown itself is approximated by a sum involving basis functions. Different unknowns may have different basis functions over an element, but a common element geometry. We therefore need a way to hold information about the basis functions associated with an unknown. This information is provided by a kind of grid overlay, called basis function grid. The basis function grid holds the nodes associated with the definition of the basis functions and a numbering of the corresponding degrees of freedom. That is, we need to store the coordinates of each basis function node and which nodes that belong to which elements (connectivity information). We also need to mark basis function nodes on the boundary. In case the element is isoparametric, no additional information about the basis function nodes need to be stored since all necessary information is available in the underlying geometry grid. In our example with quadratic triangular elements for the velocity components and linear triangular elements for the pressure, the velocities apply standard isoparametric finite elements, whereas the pressure needs a basis function grid. In a triangular element for the pressure, there are three basis function nodes, one at each corner of the element. (The geometry functions are defined in terms of six nodes.)

If the code is organized in terms of classes and objects, it is natural to have a special class for conventional finite element grids. We then introduce the basis function grid as a new class containing (i) a pointer to a conventional grid class for holding the element geometry information, and (ii) data structures for holding the basis function nodes (coordinates, connectivity, boundary markers). All information about the basis function nodes is provided through member functions in the class. In case the element is isoparametric, these member functions look up the requested information in the underlying geometry grid, otherwise they access the additional data structures.

Each unknown in the system of PDEs is represented by a field class, which holds a pointer to a basis function grid and an array of degrees of freedom, plus interpolation and other types of convenient functionality. In this way we achieve a clean design. Given a field object, we can access element information from its basis function grid, which behaves similarly to a conventional grid. 


\subsection{Programming with Mixed Finite Elements}

We have described how the information about the mixed finite elements is stored in a basis function grid associated with each unknown. When it comes to programming with mixed finite elements, we need to evaluate formulas in the integrand in the weak formulation. An example of an integrand in a mixed finite element problem may be written as $L_{i} \nabla N_{j}$, where $L_{i}$ and $N_{i}$ are different basis functions. At an integration point in an element we therefore need to evaluate the basis functions and their derivatives (with respect to physical coordinates) associated with every unknown function in the PDE system.

In a conventional finite element program, we will normally collect all information about the basis functions and the isoparametric mapping in a class, here called the FiniteElement class. Any object of this class can evaluate the basis functions and their derivatives at a given point, map local coordinates in the reference domain to the corresponding coordinates in the physical coordinate system, calculate the Jacobian of the mapping and the derivatives of the basis functions with respect to physical coordinates. With this information at hand, it is easy to evaluate expressions in the integrand in the weak form.

A mixed finite element method has in principle one type of finite element for each unknown in the system of PDEs. Any type of finite element can be represented by a FiniteElement object. Hence, to program with mixed finite elements, it is natural to introduce a new class, called MxFiniteElement, which holds a list of FiniteElement objects, one for each unknown in the PDE system. With the MxFiniteElement class coded in $\mathrm{C}++$, we can then evaluate an expression like $L_{i} \nabla N_{j}$ with the following (hopefully) self-explanatory and easily readable code:

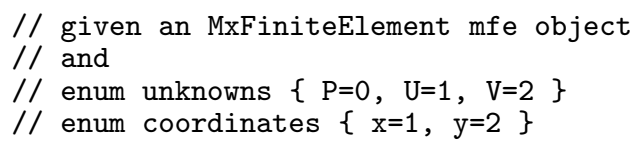

The indexing $\mathrm{mfe}(\mathrm{P})$, here meaning $\mathrm{mfe}(0)$, just returns access to a conventional FiniteElement object that holds the information about a pressure element. An MxFiniteElement object is initialized from a list of all the fields associated with all the unknowns in the system of PDEs. Each field provides a basis function grid from which the various FiniteElement objects can be constructed. Clearly, programming with MxFiniteElement is as easy as programming with a conventional FiniteElement object. Figure 1 displays an overview of the type of classes we have dealt with so far.

The generic finite element "engine" needs to loop over the (geometry) elements and integration points, and for each integration point call a problemspecific function for sampling the integrand in the weak form at this point. An MxFiniteElement object must be prepared for each (geometry) element and all its FiniteElement objects must be initialized, i.e., all basis functions and their derivatives, plus the Jacobian information, must be evaluated at the current integration point. This MxFiniteElement object is then sent to the problem-specific 
function. All the application programmer has to do is to write code as outlined above, using the MxFiniteElement object. Any programmer who is familiar with conventional finite element method in this software system will be quickly up and going with mixed finite elements in the same system since the interfaces are similar. In particular, mixed finite elements on unstructured, possible locally refined grids, represent no extra difficulty.

Our impression is that many code developers have struggled with mixed finite element in general on unstructured meshes. An indicator for this assertion is that most papers investigating various "exotic" mixed finite elements involve numerical examples on uniformly partitioned rectangular or box-shaped domains. With our suggested support for mixed finite element programming, it is straightforward to test a new element in any desired geometry.

\subsection{Adding New Mixed Elements to the Element Library}

How is a new mixed finite element actually implemented in the element library? Every element is implemented as a subclass of the ElmDef base class. This subclass is supposed to provide member functions for evaluating the geometry functions, the basis functions, their derivatives, location of geometry and basis function nodes, among other things. All information is given in the local reference coordinate system for the element. A range of conventional and mixed finite elements have been implemented in Diffpack. Some of these are: 9-node quadrilateral geometry with bilinear basis functions, 9-node quadrilateral geometry with a constant basis function, 6-node triangular geometry with linear basis functions, 6-node triangular geometry with a constant basis function, 4-node quadrilateral geometry with a constant basis function, and the similar elements in 3D. Among the non-conforming elements we find the Rannacher-Turek and the CrouzeixRaviart elements (4-node quadrilateral and 3-node triangular geometry with basis function nodes on the middle of each side and continuity at this mid-point only), the MINI element (which is a standard triangular linear element and a cubic bobble function that is zero on the element boundary), the Raviart-Thomas elements (with vector basis functions with nodes at the mid-point of each side and continuity only in the normal direction on the mid-point), the Mardal-TaiWinther [8] element (which is a non-conforming Stokes element that also works for the mixed Poisson problem, it is a cubic polynomial with 9 nodes and is continuous in the normal direction and has continuous middle value in the tangential direction). The Raviart-Thomas and the Mardal-Tai-Winther elements require special care. They are vector elements and since the standard geometry mapping does not preserve the continuity in the normal direction at each node, this mapping cannot be used. A special vector mapping needs to be used (see [1]); this mapping can also be described as a matrix mapping of the reference vector element. Since this mapping cannot be described through a FiniteElement object for scalar elements, MxFiniteElement also has the additional functionality for vector elements. This is realized such that MxFiniteElement has a pointer to a MxMapping object, which is the implementation of this special mapping. The MxMapping object is initialized and used behind the curtain if it is necessary. 
Hence, the application coder does not need to worry about these issues, but needs to change the code slightly. The above example then reads,

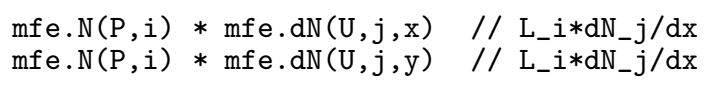

For elements that do not require this special mapping $m f e . N(P, i)$ and $m f e . d N(U, j, x)$ are identical to $\mathrm{mfe}(\mathrm{P}) . \mathrm{N}(\mathrm{i})$ and $\mathrm{mfe}(\mathrm{U}) \cdot \mathrm{dN}(\mathrm{j}, \mathrm{x})$, respectively.

\subsection{Modification of Finite Element Algorithms}

We have tried to explain how the mixed finite element software abstractions are built "on top" of conventional finite element abstractions. However, the generic algorithms for looping over elements and integration points and assembling element-level contributions must be rewritten for mixed finite elements in a strongly typed language such as $\mathrm{C}++$, because the mixed finite element classes have other names than the conventional finite element classes (i.e., the original algorithms work with FiniteElement objects, whereas the mixed versions work with MxFiniteElement). This could be avoided by parameterizing classes in the generic finite element "engine", using either templates or object orientation to hide the difference between (e.g.) FiniteElement and MxFiniteElement. We have considered this approach, but found it cleaner to duplicate finite element algorithms, mainly because the implementation of these algorithms is quite compact and because "too parameterized" template implementations might be difficult to understand and modify for a user.

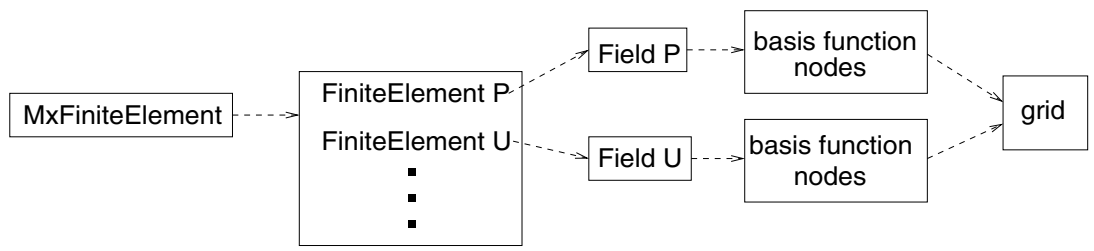

Fig. 1. Relation between central classes and objects related to the mixed elements. Dashed arrows represent pointers.

\section{Block Matrices and Block Preconditioning}

The initial version of Diffpack did not have support for block matrices. However, the standard matrix in Diffpack, called Matrix (a base class for a wide range of matrix formats), was wrapped in a class LinEqMatrix for use in linear systems. Several years later, the LinEqMatrix class was extended to handle matrices of Matrix pointers, i.e., block matrices. Similarly, the initial version of Diffpack wrapped ordinary vectors in a class LinEqVector for use in linear systems, and 
LinEqVector could easily be extended to a collection of block vectors. By adding a relatively small piece of block matrix support in LinEqMatrix and LinEqVector, the whole Diffpack system could easily exploit the block form of linear system. The coding effort was limited to implementing

- a data-structure that has a matrix of pointers to matrices,

- block-matrix-block-vector product,

- various printing, initialization and data access functionality.

Similarly, a block preconditioner,

$$
\left[\begin{array}{cc}
M & 0 \\
0 & L
\end{array}\right]
$$

is implemented as matrix of preconditioners. The needed functionality is similar to what is needed for block matrices

- a data-structure that has a matrix of pointers to preconditioners,

- block-matrix-block-vector product of preconditioners applied to vectors,

- various printing, initialization and data access functionality.

The preconditioned system reads

$$
\left[\begin{array}{cc}
M & 0 \\
0 & L
\end{array}\right]\left[\begin{array}{cc}
A & B^{T} \\
B & 0
\end{array}\right]\left[\begin{array}{l}
v \\
p
\end{array}\right]=\left[\begin{array}{cc}
M & 0 \\
0 & L
\end{array}\right]\left[\begin{array}{l}
f \\
g
\end{array}\right]
$$

Hence, we see that when using iterative methods, block matrix and block preconditioning support can be implemented relatively easy. All that is needed is to include a block-matrix-block-vector product of simpler matrices and preconditioners. These sub matrices and preconditioners are usually already implemented and tested extensively.

These outlined modifications are localized and fairly small thanks to the use abstract data types and object-oriented programming (in our case through $\mathrm{C}++$ class hierarchies). A lesson that we have repeatedly learned during the Diffpack development over the last decade, is that a flexible object-based/object-oriented design allows a package to be extended far beyond its initial application area with ease and reliability (the less code that needs to be the touch, the fewer bugs will show up later).

A lot of effort has been put into developing fast iterative methods for the indefinite problem (1). However, the knowledge of the efficiency and robustness of these methods have not yet reached the maturity level of, e.g., elliptic problems. It therefore seems natural to assume that several numerical solution strategies should be tested to find the optimal approach in each particular test case. Most solvers around use operator splitting techniques to solve (1). They are usually closely related to a basic iteration on the pressure Schur complement (2) [11]. We therefore explain briefly the basics of these methods before we see how they can be reused in the above framework. The basic steps in these solvers are, 
1. Solve $\boldsymbol{A} \boldsymbol{u}^{n}=\boldsymbol{l}$,

2. Solve $\boldsymbol{N} \boldsymbol{p}^{n}=\boldsymbol{m}$, where $\boldsymbol{N} \sim \boldsymbol{B} \boldsymbol{A}^{-1} \boldsymbol{B}$.

Here, the right-hand sides $\boldsymbol{l}$ and $\boldsymbol{m}$ depend on which algorithm that is used as the outer iteration, but they are related to the residual. The preconditioners or solvers for $\boldsymbol{A}$ and $\boldsymbol{N}$ are usually iterative solvers (like multilevel methods) that are used with some kind of convergence criterion. These solvers are, in our case and in general, solvers that have been developed, debugged and tested thoroughly, and the point is that they can often be reused without modifications. In the basic iteration on the pressure Schur complement described in [11], $\boldsymbol{A}$ have to be solved exactly, that is, $\boldsymbol{A}^{-1}$ is usually not inverted, but computed with an iterative method with sufficient accuracy to avoid affecting the outer iteration. One the other hand, $\boldsymbol{N}$ is a preconditioner and influences the efficiency of the solution algorithm only and whether it converges or not. There are also, usually, some parameters that need to be chosen and tuned numerically for optimal performance and avoiding divergence. Notice that the steps 1-2 are present in most solvers and consumes often a considerable amount of code lines. Wrapping 1-2 in suitable objects and including these objects in the sketched framework may extend existing codes with a range of solution strategies.

Alternatively, one could solve the coupled system (4) directly. The disadvantage is that the preconditioned system is still indefinite, in contrast to the pressure Schur complement, and breakdown may occur. However, if $\boldsymbol{M}$ and $\boldsymbol{L}$ are constructed properly, then the block preconditioner described in (4) bound the eigenvalue spectrum away from zero and breakdown is no longer a problem [10]. Furthermore, the components in the preconditioner (3) should be exactly the same as the ones used in the operator splitting methods,

$$
M \sim A^{-1}, \quad L \sim N^{-1}
$$

Therefore, the software developed for operator splitting schemes can be reused, basically as they are, but with an outer loop that is more robust (no need to tune parameters).

One other advantage with this approach is that $\boldsymbol{M}$ can be a cheap approximation of $\boldsymbol{A}^{-1}$, e.g, one multigrid sweep. In this case there is no need to choose an inner stop criterion that is strict enough to preserve the accuracy of the outer iteration. Since $\boldsymbol{A}^{-1}$ is usually computed by an iterative method and $\boldsymbol{L}=\boldsymbol{N}^{-1}$ already is a preconditioner, it is straightforward to utilize these solvers as preconditioners. Krylov solvers are particularly interesting as outer iterations, because there is no need to tune parameters, they "always" converge, but the convergence is sensitive to the condition number of the preconditioned system. Therefore, it is not easy to determine which approach is best in a particular application. A framework that makes the application tester capable of choosing various algorithms is desirable. 


\section{Concluding Remarks}

In this paper we have outlined how an existing finite element code can be extended to handle mixed finite elements and associated block preconditioning in an easy way. Basic objects in the conventional code, such as grids, finite elements, and scalar/vector fields, are re-used as building blocks in new objects for mixed finite elements for each unknown in the PDE system and grids with basis function information. This allows the application programming interface to the library to remain (almost) the same when mixed elements are added.

Mixed finite elements lead to indefinite matrix problems, and promising efficient solution methods exploit the block nature of the system. We have outlined the software support for block-oriented linear algebra (matrices, vectors, preconditioners) and mentioned that the the "block management" is merely a top layer in a package. That is, exploiting the block structure requires just some managing software at a much higher abstraction level than the number crunching loops. Much of the software pieces needed in block-oriented algorithms are classical numerical strategies already available in many codes.

\section{References}

1. F. Brezzi and M. Fortin. Mixed and Hybrid Finite Element Methods. Springer, 1991.

2. P. M. Gresho and R. L. Sani. Incompressible Flow and the Finite Element Method. Wiley, 1998.

3. Internet. Diffpack software package. http://www.diffpack.com.

4. H. P. Langtangen. Computational Partial Differential Equations - Numerical Methods and Diffpack Programming. Lecture Notes in Computational Science and Engineering. Springer, 1999.

5. H. P. Langtangen, K.-A. Mardal, and R. Winther. Numerical methods for incompressible viscous flow. Advances in Water Resources, 2002. submitted.

6. K.-A. Mardal and H. P. Langtangen. Mixed finite elements. In H. P. Langtangen and A. Tveito, editors, Computational Partial Differential Equations using Diffpack - Advanced Topics. Springer, 2002. In preparation.

7. K.-A. Mardal, J. Sundnes, A. Tveito, and H. P. Langtangen. Block preconditioning for systems of PDEs. In H. P. Langtangen and A. Tveito, editors, Computational Partial Differential Equations using Diffpack - Advanced Topics. Springer, 2002. In preparation.

8. K.-A. Mardal, X.-C. Tai, and R. Winther. Robust finite elements for Darcy-Stokes flow. (Submitted), 2001.

9. R. Rannacher. Finite element methods for the incompressible Navier-Stokes equation. 1999.

http://www.iwr.uni-heidelberg.de/sfb359/Preprints1999.html.

10. T. Rusten and R. Winther. A preconditioned iterative method for saddlepoint problems. SIAM J. Matrix Anal., 1992.

11. S. Turek. Efficient Solvers for Incompressible Flow Problems. Springer, 1999. 